\title{
Primary Hyperparathyroidism in a Twin Pregnancy: A Case Report
}

\author{
Luis Eduardo Pérez Sánchez, Julio César Jordan Balanza, Alicia Goya Pacheco, \\ Pilar Elena González de Chaves Rodríguez, Manuel Barrera Gómez \\ Endocrine Surgery Unit, Department of General and Digestive Surgery, Hospital Universitario Nuestra Señora \\ de Candelaria, Tenerife, Spain \\ Email: I.eduardopesan@gmail.com
}

Received 20 May 2016; accepted 12 June 2016; published 15 June 2016

Copyright @ 2016 by authors and Scientific Research Publishing Inc.

This work is licensed under the Creative Commons Attribution International License (CC BY). http://creativecommons.org/licenses/by/4.0/

c) (i) Open Access

\begin{abstract}
The primary hyperparathyroidism during pregnancy is an infrequent disorder but it is rarer in a twin gestation. Often it is not suspected because the symptomatology is overlapped with the normal course of pregnancy and it is usually discovered after a routine blood test. Ultrasound is the best option to identify the adenoma, while the gold-standard is technetium-99 m sestamibi but is contraindicated in this situation. The treatment is controversial and depends of the time of the gestation: the medical therapy is accepted in the first and third trimester, and the surgery is reserved for the second trimester, where the side effects are low. We present a case of primary hyperparathyroidism in a twin pregnancy treated by a minimally invasive parathyroidectomy in the second trimester without consequences for both, mother and newborns.
\end{abstract}

\section{Keywords}

Hyperparathyroidism, Pregnancy, Surgery

\section{Introduction}

Primary hyperparathyroidism (PHPT) is a common entity characterized by elevated production of parathyroid hormone (PTH) and hypercalcemia [1]. This is the third most common endocrine disorder and the first cause of hypercalcemia in outpatients with an incidence of 20/100,000 habitants/year and a prevalence of more than 100,000 new cases in United State [2]. A single adenoma is observed in approximately in the $80 \%$ of the cases of this pathology [3]. PHPT during pregnancy, however, is a rare entity that is only described in few cases in the literature, and it is even rarer in a twin pregnancy.

Often it is difficult to recognize the PHPT during pregnancy because the physiological changes occult the 
signs and symptoms and could be misdiagnosed [4]. In the other hand, the symptoms of PHPT commonly are confused with the normal development of the pregnancy such as abdominal pain, vomiting, nausea, fatigue, headaches, confusion and irritability [5]. To confirm the diagnosis it is necessary to test out elevated serum calcium and PTH levels, and the ultrasounds are the best option to localize the illness parathyroid [6].

The best option of treatment in these cases is the surgery, preferably in the second trimester [7]. Maternal and fetal complications are frequents if the treatment is not accomplished, and untreated disease could commonly complicate fetal development and mortality [8].

We present a rare case of PHPT in a twin gestation, successfully treated with a minimally invasive parathyroidectomy. To our knowledge, there are less than 5 cases described in the literature.

\section{Case Report}

A 28-year-old tertigravida (G3P1A1) woman was first evaluated for hyperemesis and hypertension during her $12^{\text {th }}$ week of biamniotic-gestation. Her previously pregnancy ended without remarkable incidences. She had history of various metatarsians fracture and sprains and an abortion before her first pregnancy. She got a hypothyroidism which was controlled without medical treatments. Her family history did not reveal any case of hypercalcemia or endocrine tumors. Metildopa, cianocobalamin, folic acid and iodine were her medical treatment before the surgery.

The patient was well nourished and in no distress. Her blood pressure was 144/109 mmHg, heart rate 75 bpm and body mass index (BMI) of 23.2. The examination of her neck was normal. On the abdominal exploration she presented normal measures for a 12 th week bi-gestation. The remainder of the systemic examination was unremarkable.

The initial laboratory evaluation showed an important elevation of calcium level of $13.2 \mathrm{mg} / \mathrm{dl}$ (reference range 8.1 - 10.5) and normal phosphate level of $2.7 \mathrm{mg} / \mathrm{dl}$ (reference range 2.50 - 4.50). Her hormones analysis revealed a diminished thyrotropin levels of $0.160 \mu \mathrm{UI} / \mathrm{ml}$ (reference range $0.270-4.200$ ) with normal values of T3 and T4 hormones, and PTH level was $78.6 \mathrm{pg} / \mathrm{ml}$ (reference range 10 - 65). Renal function was normal with an elevation of urinary calcium level of $646.8 \mathrm{mg} / 24 \mathrm{hr}$ (reference range 50 - 300). With these dates of elevated PTH and urinary calcium level we made the diagnosis of PHPT and excluded others causes of hypercalcemia.

An ultrasound neck exploration showed a nodular hypoechoic lesion in the lower right pole of the thyroid gland, measuring $1.4 \times 0.9 \mathrm{~cm}$, hypervascularized, compatible with a parathyroid adenoma (Figure 1).

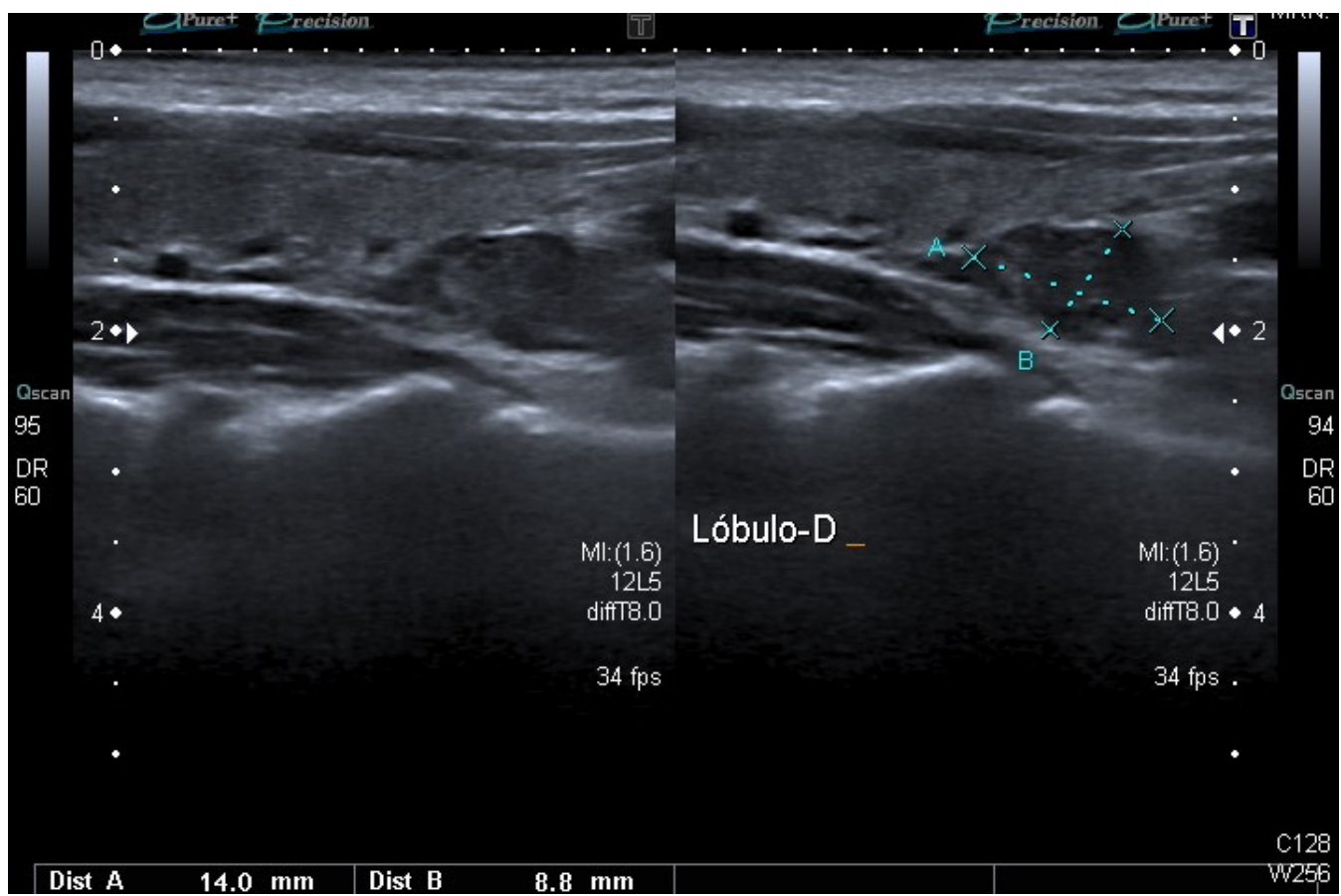

Figure 1. Cervical ultrasound. Right inferior parathyroid. 
She was evaluated by an endocrine, a gynecological and a surgery specialist and they recommended an intensive hydration, blood pressure control and programmed surgery, which was performed on the 13th week of gestation. She underwent to a minimally invasive left inferior parathyroidectomy and an adenoma of $1.5 \mathrm{~cm}$ was removed without complications (Figure 2 and Figure 3). The levels of PTH during the surgery after removing the

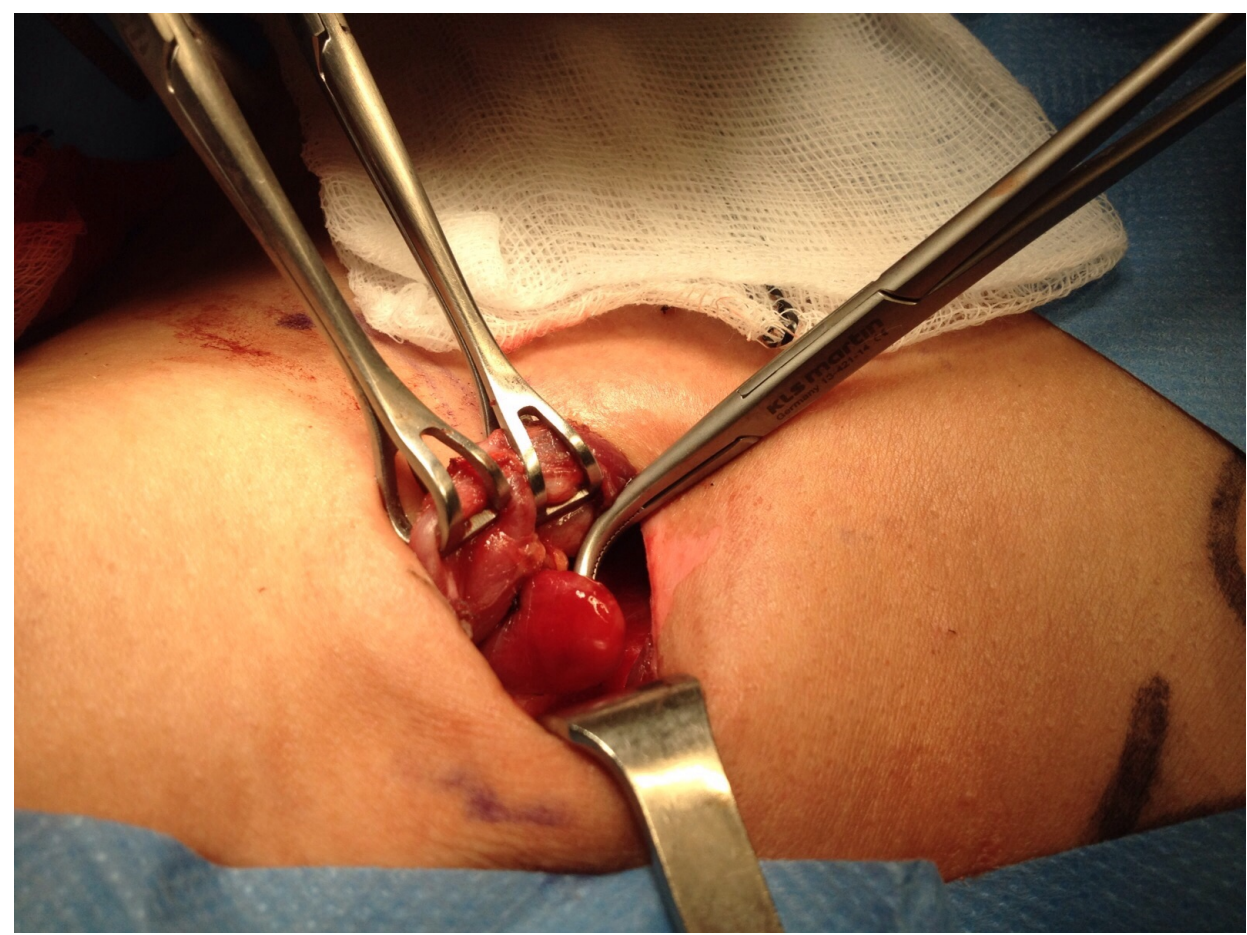

Figure 2. Right inferior minimally invasive parathyroidectomy.

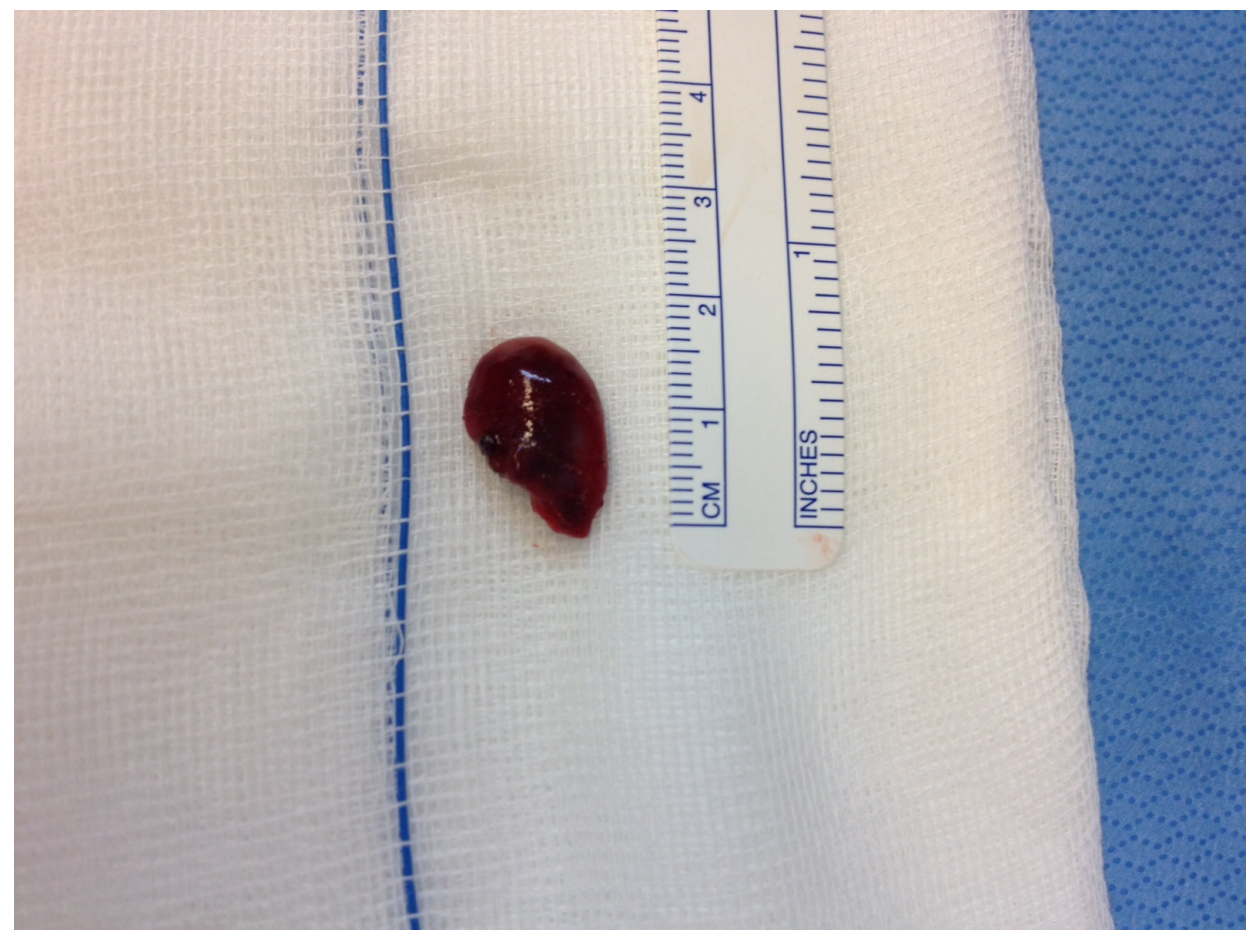

Figure 3. Right inferior parathyroid. 
adenoma decreased more than an $80 \%$ respect to pre-incision level: PTH basal $345.5 \mathrm{pg} / \mathrm{ml}$, PTH pre-incision $520.4 \mathrm{pg} / \mathrm{ml}$ and PTH 10 minutes after excision $80.7 \mathrm{pg} / \mathrm{ml}$. With these values we confirmed that the enlarged parathyroid was the pathological adenoma. Post-operation PTH and calcium level come to normal values (43.9 $\mathrm{pg} / \mathrm{ml}$ and $9 \mathrm{mg} / \mathrm{dl}$, respectively). Histopathological exams confirmed the hypothesis with a parathyroid adenoma that weighing $73 \mathrm{~g}$.

During the postoperative, the patient evolved satisfactorily and was discharged on the fourth day after the surgery. In her home she was asymptomatic, remained nor mocalcemic and the pregnancy continued until her delivery on $35^{+5}$ weeks. Two males were born by a caesarean section, with normal weight and height, and blood tests showed normal serum values of calcium (Newborn 1: weight $2530 \mathrm{~g}$; height $43 \mathrm{~cm}$; serum $\mathrm{Ca}^{+2} 8.8$ mg/dl/Newborn 2: weight $1967 \mathrm{~g}$; height $44 \mathrm{~cm}$; serum $\mathrm{Ca}^{+2} 8.9 \mathrm{mg} / \mathrm{dl}$ ). During the following, both mother and newborns did not develop complications or changes in their calcium or phosphate levels.

\section{Discussion}

PHPT in pregnancy needs to be recognized and treated due to the risks it confers to both, mother and foetus [6]. The prevalence of PHPT during pregnancy is unknown. There are less than 200 cases described in the literature. In many cases, an unexplained hypercalcemia as an incidental finding in a routine blood analysis is the first data to suspect a PHPT [9]. In normal pregnancy, the intestinal absorption of calcium is increased resulting in a state of absorptive hypercalciuria. Serum levels of PTH are low in the first half of pregnancy and increased towards the end of pregnancy. During the gestation, there is an increase of serum albumin in the extracellular fluid volume and a decrease in the concentration. Thus, total serum calcium is low in pregnancy, but ionized calcium is normal. Some of these changes may mask the degree of hypercalcemia that might, otherwise, be more apparent in non-pregnant women [10]. Also, the symptoms of PHPT, such as hyperemesis, preeclampsia, abdominal pain, fatigue or irritability [5] [8], can be similar to those produced by the pregnancy itself, and masked this pathology.

Maternal complications can occur in up to $67 \%$ of untreated patients and included gastrointestinal symptoms, nephrolithiasis, pancreatitis, hyperemesis gravidarum, the HELLP syndrome and pre-eclampsia [8] [10]. Another complication can be a spontaneous abortion, and this happened to our patient. The delivery is a critical moment for the mothers because a hypercalcemic crisis can occur with a fatal outcome [5]. Fetal complications in untreated PHPT mothers can affect to $80 \%$ of newborns including intrauterine growth restriction, low birth weight, preterm delivery and intrauterine fetal demise [11]. After the delivery, the most dangerous complication is neonatal hypocalcemia, more frequently it is transient but in a few cases can be permanent [11].

Diagnosis of PHPT is classically based upon laboratory findings of elevated serum calcium with an inappropriately elevated PTH [9]. In approximately $80 \%$ of cases, a single adenoma is the cause of PHPT [3]. To confirm the localization, a parathyroid image is necessary and the ultrasound has an elevated sensibility and specificity without radiation [5] [7] [9]. However, the gold standard for the diagnosis of PHPT is the technetium-99 m sestamibi but it is contraindicated during the pregnancy because it presents a risk of radiation for the foetus. If ultrasound fails to localize the adenoma, a magnetic resonance would be safe [7] [8].

The optimal management of hyperparathyroidism in pregnancy has been debated in the literature, especially in cases diagnosed in the first or third trimester [12]. The treatment should be individualized based on severity, symptoms, gestational age, medical condition and the reliability of the mother [8]. Conservative therapy, with hydration and phosphates, is accepted in asymptomatic and mild hypercalcemia based in the risks of anesthesia and surgery during the pregnancy [6] [12]. However, the significant maternal, fetal and neonatal risks associated with maternal PHPT, and the improved safety of general anesthesia during pregnancy, surgical management has been increasingly recommended [8]. The optimal time for surgery has generally been considered the second trimester [5]-[7] [12], while management of newly recognized clinically significant hypercalcemia in the third trimester is under debate [10]. If the diagnosis is made in the third trimester, maternal and fetal factors should be weighed against possible risks of surgery [5].

Classically, the bilateral neck exploration (BNE) has been the gold standard in the surgery of parathyroid. However, the development of new imaging techniques has allowed us to do a more accurate localization of the parathyroid illness and to avoid a large incision in the neck. A minimally invasive parathyroidectomy (MIP) technique has proven to reduce pain, improve cosmetic and achieve a similar cure rate when compared to the BNE [12]. Even, a video assisted-MIP with bilateral neck exploration is an option when, by imaging, we do not have localized the pathology gland [7]. 
Actually, the preference for surgery over medical management has been well established. Neonatal complication rates are $37 \%$ after medical treatment and only $10 \%$ after surgical intervention [5]. In the other hand, it is not possible to predict complications, in maternal or fetal terms, based on calcium levels. Moreover, surgery offers the possibility of definitive treatment with a low risk for the fetus and mother [10] [12]. For this reason, we decided to perform surgery in our case, with an unremarkable recovery for the mother and her newborns.

In our case, when we diagnosed the PHPT, we performed a literature review and considered that the surgical treatment, by a MIP technique, was the best choice for the patient and the newborns. The second trimester was the right moment to perform the surgery according to the literature and the ultrasound was enough to confirm the localization of the adenoma. We believe that the benefit of the surgical treatment was justified and we managed to treat the disease definitively with a minimal risk for both, mother and newborns.

\section{Conclusion}

The PHPT during a pregnancy is an uncommon entity and very rare in a twin gestation. Suspecting is important to do the diagnosis when we have an unexplained hypercalcemia. The conservative management should be applied in specific cases. By contrast, in our opinion, the surgical approach, principally in the second trimester and through a MIP, should be the base of the treatment.

\section{Patient Consent}

We have obtained the consent of the patient for publication of this case.

\section{References}

[1] Silverberg, S.J. and Bilezikian, J.P. (2013) Primary Hyperparathyroidism: Pathophysiology and Surgical Indications, Work-Up. In: Randolph, G.H., Eds., Surgery of Thyroid and Parathyroid Glands, 2nd Edition, Chapter 56, John Wiley \& Sons, Philadelphia, 531-538. http://dx.doi.org/10.1002/9781118453926.ch68

[2] Lal, G. and Clark, O.H. (2005) Diagnosis of Primary Hyperparathyroidism and Indications for Parathyroidectomy. In: Clark, O.H. and Duh, Q.-Y., Eds., Textbook of Endocrine Surgery, 2nd Edition, Chapter 40, Elsevier, Philadelphia, 384-392. http://dx.doi.org/10.1016/b978-0-7216-0139-7.50044-2

[3] MacKenzie-Feder, J., Sirrs, S., Anderson, D., Sharif, J. and Khan, A. (2011) Primary Hyperparathyroidism: An Overview. International Journal of Endocrinology, 2011, Article ID: 251410. http://dx.doi.org/10.1155/2011/251410

[4] Abood, A. and Vestergaard, P. (2014) Pregnancy Outcomes in Women with Primary Hyperparathyroidism. European Journal of Endocrinology, 171, 69-76. http://dx.doi.org/10.1530/EJE-13-0966

[5] Hession, P., Walsh, J. and Gaffney, G. (2014) Two Cases of Primary Hyperparathyroidism in Pregnancy. British Medical Journal of Case Reports, 2013, 1-5. http://dx.doi.org/10.1136/bcr-2013-202883

[6] Naru, T., Khan, R.S. and Khan, M.A. (2011) Primary Hyperparathyrodism in Pregnancy and Review of Literature. The Journal of the Pakistan Medical Association, 61, 401-403.

[7] Bendinelli, C., Nebauer, S., Quach, T., Mcgrath, S. and Acharya, S. (2013) Is Minimally Invasive Parathyroid Surgery an Option for Patients with Gestational Primary Hyperparathyroidism? BioMed Central Pregnancy and Childbirth, 13, 1-6. http://dx.doi.org/10.1186/1471-2393-13-130

[8] Truong, M.T., Lalakea, M.L., Robbins, P. and Friduss, M. (2008) Primary Hyperparathyroidism in Pregnancy: A Case Series and Review. Laryngoscope, 118, 1966-1969. http://dx.doi.org/10.1097/MLG.0b013e318180276f

[9] Walker, A., Jimeno Fraile, J. and Hubbard, J.G. (2014) Parathyroidectomy in Pregnancy: A Single Centre Experience with Review of Evidence and Proposal for Treatment Algorithm. Gland Surgery, 3, 158-164.

[10] Pothiwala, P. and Levine, S.N. (2009) Parathyroid Surgery in Pregnancy: Review of the Literature and Localization by Aspiration for Parathyroid Hormone Levels. Journal of Perinatology, 29, 779-784. http://dx.doi.org/10.1038/jp.2009.84

[11] Herrera-Martínez, A.D., Bahamondes-Opazo, R., Palomares-Ortega, R., Muñoz-Jiménez, C., Gálvez-Moreno, M.A. and Quesada Gómez, J.M. (2015) Primary Hyperparathyroidism in Pregnancy: A Two-Case Reports and Literature Review. Obstetrics and Gynecology, 2015, Article ID: 171828. http://dx.doi.org/10.1155/2015/171828

[12] McMullen, T.P.W., Learoyd, D.L., Williams, D.C., Sywak, M.S., Sidhu, S.B. and Delbridge, L.W. (2010) Hyperparathyroidism in Pregnancy: Options for Localization and Surgical Therapy. World Journal of Surgery, 34, 1811-1816. http://dx.doi.org/10.1007/s00268-010-0569-2 